\title{
Tracheoesophageal fistula as the presenting manifestation of Hodgkin's lymphoma
}

\section{David Alba, Salvador Díaz Lobato ${ }^{1}$, Rodolfo Alvarez-Sala ${ }^{1}$, Carlos Villasante $^{1}$ and Celina Echevarría ${ }^{2}$} Departments of Internal Medicine, ${ }^{1}$ Respiratory Medicine and ${ }^{2}$ Pathology, Hospital 'La Paz', Madrid,
Paseo de la Castellana 261, 28046-Madrid, Spain

\begin{abstract}
Summary: We present a patient with tracheoesophageal fistula as the initial manifestation of Hodgkin's disease with oesophageal involvement. To our knowledge, this has not been previously reported. The diagnosis of Hodgkin's lymphoma was made at autopsy.
\end{abstract}

\section{Introduction}

Tracheoesophageal fistula (TEF) in adult patients is reported mainly in carcinoma of the lung or oesophagus. ' Lymphoma rarely involves the oesophagus. ${ }^{2}$ The occurrence of a TEF in a patient with lymphoma is even more unusual; most cases have been documented in patients who have undergone radiation therapy or chemotherapy. ${ }^{3}$

We present a case of Hodgkin's disease with involvement of the oesophagus who had TEF at presentation. To our knowledge, this has not been previously reported.

\section{Case report}

A 60 year old woman presented with fever and productive cough of 20 days' duration. She was treated with antibiotics by her local physician without improvement. One day prior to admission the patient developed dyspnoea, and she was noted to cough after swallowing liquids. There was a history of hypertension for which she had diuretic therapy.

On physical examination the patient was an overweight female who was alert and oriented. Her blood pressure was $210 / 130 \mathrm{mmHg}$, pulse rate was $90 /$ minute, respiration 28 /minute and temperature $38.5^{\circ} \mathrm{C}$. No lymphadenopathy or increased jugular venous pressure was found. Diffuse crackles were heard in the lower third of both lung fields. The heart rate was irregular, with a grade 2 systolic murmur. There was peripheral oedema.

Results of laboratory studies were remarkable for a white blood count of $5.3 \times 10^{9} / 1$ with $89 \%$

Correspondence: D.A. Martín, M.D., C/ Isabel Rosillo, 8, $1^{\circ} \mathrm{C}$, Alcobendas, 28100-Madrid, Spain.

Accepted: 23 June 1993 neutrophils, haematocrit of $36 \%$, lactic dehydrogenase of $520 \mathrm{U} / 1$ (normal range 230-460 U/1), and fibrinogen of $12.28 \mathrm{~g} / \mathrm{l}$ (normal range $1.5-3.5 \mathrm{~g} / \mathrm{l}$ ). Arterial blood gas on room air showed a $\mathrm{pH}$ of 7.48, $\mathrm{PCO}_{2} 34.6 \mathrm{mmHg}$ and $\mathrm{PO}_{2} 58 \mathrm{mmHg}$. Blood and urine cultures were negative. No acid-fast bacilli or fungi were seen on sputum specimens, and culture of sputum yielded a Gram-negative mixed flora. An electrocardiogram demonstrated atrial fibrillation at rate of 90 . Chest X-ray showed right lower lobe alveolar infiltrates.

The patient was treated with intravenous erythromycin and frusemide, with moderate improvement in the first day. However, she developed a bout of respiratory failure that required intubation and mechanical ventilation. Chest X-ray showed bilateral diffuse alveolar infiltrates, compatible with severe respiratory distress syndrome.

At the moment of intubation, purulent material flowed through the endotracheal tube, and with mechanical breaths the air leaked through a nasogastric probe. A tracheoesophageal fistula was

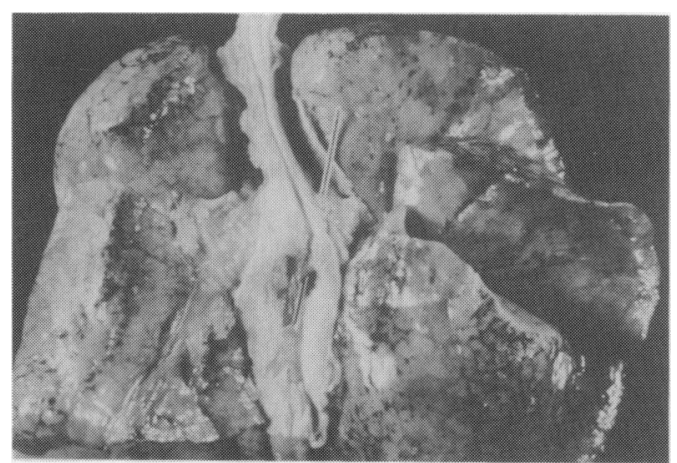

Figure 1 Fistula between rigt mainstem bronchus and oesophagus. 
suspected, and bronchoscopy visualized right mainstem bronchus perforation, surrounded by granulomatous tissue. In spite of mechanical ventilation, respiratory status deteriorated and the patient died on the seventh hospital day.

Autopsy showed a fistula between right mainstem bronchus and oesophagus (Figure 1) and mediastinal mass. Microscopic examination revealed mixed cellularity Hodgkin's disease involving mediastinal lymph glands, the lower third of the oesophagus and retroperitoneal lymph glands.

\section{Discussion}

Oesophageal involvement by lymphoma either as a primary disease or as manifestation of disseminated disease is distinctly uncommon. ${ }^{2,4}$ In a review of gastrointestinal lymphoma comprising 117 cases, no oesophageal involvement was found. ${ }^{5}$ Likewise, oesophageal involvement with Hodgkin's disease has been reported infrequently. ${ }^{6.7}$

The term TEF is used to refer any fistulization between the oesophagus and trachea or the lung, regardless of the origin of the lesion, including broncho-oesophageal fistulas. ${ }^{1,3} \mathrm{~A}$ total of 40 patients with lymphoma and TEF have been reported ${ }^{3}$ ( $85 \%$ had Hodgkin's disease). Only ten patients had the TEF before any therapy. The fistula was located between the oesophagus and trachea in 29 patients, the oesophagus and left mainstem bronchus in 10 patients, and between the

\section{References}

1. Daranceau, A. \& Jamieson, G.G. Malignant tracheoesophageal fistula. Ann Thorac Surg 1984, 37: 346-354.

2. Ehrlich, A.N., Stalder, G., Geller, W. \& Sherlock, P. Gastrointestinal manifestations of malignant lymphoma. Gastroenterology 1968, 54: 1115-1121.

3. Perry, R.R., Rosenberg, R.K. \& Pass, H.I. Tracheoesophageal fistula in the patient with lymphoma: case report and review of the literature. Surgery 1989, 105: 770-777.

4. Agha, F.P. \& Schnitzer, B. Esophageal involvement in lymphoma. Am J Gastroenterol 1985, 80: 412-416.

5. Lewin, K.J., Ranchod, M. \& Dorfman, R.F. Lymphoma of the gastrointestinal tract. Cancer 1978, 42: 693-697.

6. Jackson, H. \& Parker, F. Hodgkin's disease: IV. Involvement of certain organs. $N$ Engl J Med 1945, 232: 547-560. oesophagus and lobar bronchus in one patient. No cases have been reported previously between the $c$ oesophagus and right mainstem bronchus. TEF commonly results from lymphoma that originates in the mediastinal lymph nodes. ${ }^{8.9}$

Development of TEF in the setting of malignancy is generally a poor prognostic event. 'Aspiration pneumonia is the immediate cause of death in the greater proportion of patients. The only \& effective treatment is to exclude the fistula from the alimentary tract. This may be achieved by intubation or operation. ${ }^{1}$ The development of TEF in the patients with lymphoma is also a serious event. ${ }^{10,11}$ However, these patients have a better prognosis than do patients with primary carcinoma of the oesophagus or lung. Most of the late deaths in patients in whom the fistula was closed successfully were due to recurrent lymphoma rather than complications of TEF. ${ }^{3}$

There is no major difference in prognosis between patients who initially have TEF and are then found to have lymphoma and those who develop TEF durimg or after initial lymphoma therapy. ${ }^{3}$ In the present case, however, the acute TEF presentation with no other evidence of Hodgkin's lymphoma, prevented antemortem diagnosis and therapy.

TEF may be the presenting manifestation of Hodgkin's disease. Since long survival following treatment for Hodgkin's disease is common, early recognition and repair of TEF in these patients can be lifesaving.

7. Morrison, F.S., Critz, G., Tatum, W.T. \& Strauss, H.K Hodgkin's disease of the esophagus: successful treatment of a rare complication. Cancer 1973, 31: 1244-1246.

8. West, W.O. \& Bouroncle, B.A. Spontaneous perforation of the esophagus in Hodgkin's disease: report of three cases and literature review. Am J Gastroenterol 1960, 33: 335-342.

9. Kelly, J.P. \& Roper, L.L. Treatment of malignant tracheoesophageal fistula due to histiocytic lymphoma. South Med J 1984, 77: 1203-1205.

10. Zenny, J.C., Grenier, Ph., Favier, H., Bernard, J.F. \& Nahum, H. Fistule oeso-trachéale révélatrice d'une maladie de Hodgkin. J Radiol 1981, 62: 191-195.

11. Renzi, G. \& Lesage, R. Endobronchial Hodgkin's disease and bronchoesophageal fistula. Chest 1972, 61: 696-697. 